\title{
Serum microRNAs as novel biomarkers for early prediction of disease severity in patients with acute pancreatitis
}

Yu-Ping Zhang ${ }^{1 \dagger}$, Lei Ye ${ }^{2+}$, Na Yu ${ }^{3}$, Cheng Wang ${ }^{4,5}$, Lin Wu ${ }^{2}$, Chun-Ni Zhang ${ }^{4,5^{*}}$, Chen-Yu Zhang ${ }^{5^{*}}$ and Fang-Yu Wang ${ }^{2 *}$

\begin{abstract}
Background: Dysregulated microRNAs (miRNAs) have been identified to be associated with various diseases. However, the relationship between serum miRNAs and the severity of acute pancreatitis (AP) remains unknown.

Methods: One hundred twenty-five patients were enrolled and classified into mild AP (MAP, $n=45$ ), moderate severe AP (MSAP, $n=42$ ) and severe AP (SAP, $n=38$ ) groups according to Atlanta 2012. TaqMan low density array (TLDA) technology was initially used in three pooled serum samples from 10 MAP, 10SAP and 10 healthy controls $(\mathrm{HCs})$. The selected miRNAs were subsequently measured individually using quantitative real-time polymerase chain reaction (qRT-PCR) assay.

Results: The TLDA identified 395 miRNAs were differentially expressed between the AP patients and the HCs, among which 12 miRNAs were selected for further evaluation. qRT-PCR confirmed that miR-19a, miR-143 and miR374-5p were significantly upregulated in AP patients $(p<0.001)$ and increased with disease severity $(p<0.05)$. Receiver operating characteristic (ROC) curve analysis revealed that three miRNAs could distinguish the SAP patients from the HCs (area under ROC, AUC 0.940-0.943), MAP (AUC 0.754-0.782), and moderate severe AP (MSAP, AUC 0.670-0.686). In addition, multivariate logistic regression revealed that increased serum miR-143 was an independent predictor of developing SAP among MSAP and SAP patients $(\mathrm{OR}=6.8,95 \% \mathrm{Cl}$ 2.0-22.7) and among all AP patients (OR $=4.5,95 \% \mathrm{Cl} 1.8-10.9)$.
\end{abstract}

Conclusions: These data indicate that an expression signature of three serum miRNAs holds potential as a novel biomarker for the early prediction of SAP.

Keywords: Acute pancreatitis, miR-143, miR-19a, miR-374-5p, Early prediction

\footnotetext{
*Correspondence: zchunni27@hotmail.com; cyzhang@nju.edu.cn; wangfy65@nju.edu.cn

${ }^{\dagger}$ Yu-Ping Zhang and Lei Ye contributed equally to this work.

${ }^{4}$ Department of Clinical Laboratory, Jinling Hospital, \#305 East Zhongshan

Road, Nanjing 210002, China

${ }^{5}$ State Key Laboratory of Pharmaceutical Biotechnology, Nanjing Advanced Institute for Life Sciences (NAILS), Nanjing University School of Life Sciences, Jiangsu Engineering Research Center for microRNA Biology and Biotechnology, \#163XianlinRoad, Nanjing 210002, China

2Department of Gastroenterology and Hepatology, Jinling Hospital, Medical School of Nanjing University, \#305 East Zhongshan Road, Nanjing 210002,

China

Full list of author information is available at the end of the article
}

(c) The Author(s). 2020 Open Access This article is licensed under a Creative Commons Attribution 4.0 International License, which permits use, sharing, adaptation, distribution and reproduction in any medium or format, as long as you give appropriate credit to the original author(s) and the source, provide a link to the Creative Commons licence, and indicate if changes were made. The images or other third party material in this article are included in the article's Creative Commons licence, unless indicated otherwise in a credit line to the material. If material is not included in the article's Creative Commons licence and your intended use is not permitted by statutory regulation or exceeds the permitted use, you will need to obtain permission directly from the copyright holder. To view a copy of this licence, visit http://creativecommons.org/licenses/by/4.0/. 


\section{Introduction}

Acute pancreatitis (AP) is an inflammatory disorder of the pancreas with highly variable clinical manifestations and attacks 13 to 45 per 100, 000 people every year [1]. Although most of the patients manifest a self-limiting course, $10-20 \%$ of them may experience a severe process with a high mortality reaching up to $30-50 \%$ in cases with severe AP (SAP) [1]. Approximately $50 \%$ of deaths occur within 1 week of the attack, mostly from multiorgan dysfunction syndrome [2]. Early prediction of disease development is fundamental to determine intensive treatment to improve outcomes.

There are some clinic biochemical scoring systems, such as APACHE II and the Ranson system. However, they are too complex to keep them from being routinely applied. Currently, the widely accepted 'gold standard' for classification of the severity of AP is the Atlanta 2012 classification, and persistent organ failure (POF) has been recognized as the critical factor in determining the severity of AP. Nevertheless, patients with POF cannot be identified until $48 \mathrm{~h}$ after admission [3]. Therefore, novel biomarkers for predicting early SAP are still urgently needed.

MicroRNAs (miRNAs), a class of endogenous small non-coding RNAs has been demonstrated to be involved in multiple physiological and pathological processes. The value of miRNAs in AP has been reported in recent years. Meng $S$ et al. performed a study to confirm that serum miR-24 could well distinguish AP patients from $\mathrm{HCs}$, but there was no significant difference between mild AP (MAP) and moderately severe AP (MSAP) [4]. Serum miR-216a was reported to be a potential biomarker for SAP, but the result was retrieved from a small cohort study of subjects [5]. Lu et al. comfirmed that a cluster of miRNAs (miR-7, miR-9, miR-122 and miR-141) were increased in AP patients, and particularly, the level of miR-7 was significantly higher in SAP than that in MAP patients [6]. Moreover, these 4 serum miRNAs declined along with the clinical improvement of AP patients, which indicated that serum miRNAs might take part in the occurrence, development, and repair of AP [6]. The above findings suggest that levels of serum miRNAs in AP are different in accordance with the severity of AP, thus serum miRNAs are potential biomarkers for predicting SAP.

We hypothesized that serum miRNAs profiles may be different in MAP, MSAP and SAP patients according to Atlanta 2012 classification and could provide prognostic information at the time of diagnosis. Therefore, we conducted this case-control study to compare the profiles of serum miRNAs in the early phase of AP, with an attempt to explore whether miRNAs may serve as markers to distinguish SAP from MAP and MSAP on admission. We found that an expression signature of serum miR- 19a, miR-143 and miR-374-5p might be a potential biomarker for the early prediction of SAP.

\section{Materials and methods Study population}

Patients admitted to Jinling Hospital (Nanjing, China) within $48 \mathrm{~h}$ of AP onset from March 2014 to March 2015 were enrolled. AP was diagnosed when the patients met at least 2 of the following 3 criteria: 1 ) characteristic abdominal pain; 2) serum amylase $\geq 3$ times the upper limit of normal; 3 ) characteristic findings on abdominal imaging [7]. All enrolled subjects were recruited within $24 \mathrm{~h}$ of admission. The AP severity was defined according to Atlanta 2012 [3]. Control subjects were recruited from the Health Examination Center of Jinling Hospital. Controls were matched to the patients by age, sex and ethnicity. All subjects were provided written informed consent. The study was approved by the ethics committee of Jinling Hospital in accordance with the Declaration of Helsinki.

\section{Study design}

We included 125 patients with AP (45 with MAP, 42 with MSAP, and 38 with SAP) and 52 HCs in our study according to the above inclusion criteria. To identify early predicting markers, a multiphase case-control study was designed. In the initial screening or discovery phase, total RNA extracted from three pooled serum samples of 10 patients with MAP, 10 with SAP and 10 healthy controls were separately subjected to miRNA profiling analyses using the TaqMan low density array (TLDA) analysis, which contains probes for $754 \mathrm{miR}$ NAs. To identify serum miRNAs with prediction potential, miRNAs meeting the following four criteria were selected: First, a candidate miRNA must be differentially expressed (fold change $\geq 2$ ) between AP patients and HCs. Second, the trend of alteration between AP patients and controls must be the same as that between the SAP and MAP groups. Third, miRNAs with greater fold changes between the different groups are preferred. Finally, miRNAs expressed with high abundance in serum are preferred. The selected miRNAs were then confirmed using quantitative real-time polymerase chain reaction (qRT-PCR), in the following 2 sets: (a) the biomarker training set, including 20 MAP, 20 MSAP, 20 SAP patients and $20 \mathrm{HCs}$ (including samples that had undergone TDLA) and (b) the validation set, which comprised serum samples from an additional 25 MAP, 22 MSAP, 18 SAP patients and 32 HCs (Fig. 1).

\section{Data and sample collection}

Clinical and laboratory data were obtained from medical records. Patients' blood samples were drawn on 


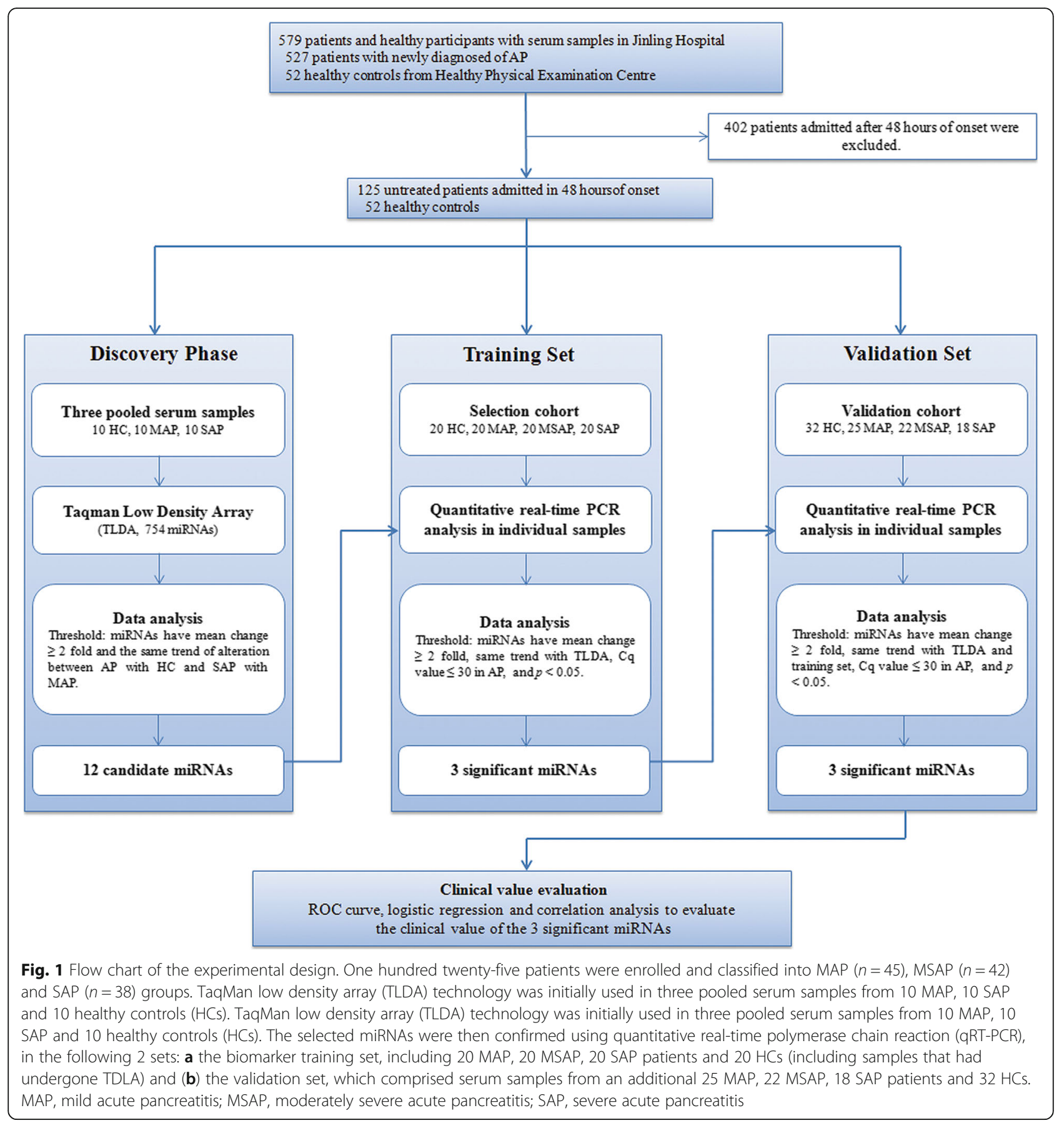

admission (day 1) and at days 2, 3, 7, 14 and 21. Within $2-3 \mathrm{~h}$ of collection, the blood samples were first centrifuged at $1500 \mathrm{~g}$ for $10 \mathrm{~min}$ (min), and the serum samples were transferred to a new tube and subjected to centrifuge at $12,000 \mathrm{~g}$ for $5 \mathrm{~min}$ to fully remove the cell debris. The supernatant was collected and stored at $-80^{\circ} \mathrm{C}$ until use.

Patients were monitored for the severity of AP. The APACHE II and Ranson scores of the patients were calculated as previously described [3], and OF identified at any time point during hospitalization was noted.

Clinical outcome included mortality, intensive care unit (ICU) admission, need for interventions, ICU length of stay (LOS), and overall hospital LOS. Interventions were defined as surgical (cystgastrostomy, open and laparoscopic pancreatic debridement), interventional (percutaneous drainage) and supportive (continuous renal replacement therapy and endotracheal intubation with artificial ventilation). 
TaqMan low density array assay (TLDA)

For the TLDA assay, total RNA was extracted from 3 pooled serums, using TRIzol reagent (Invitrogen, Carlsbad, CA, USA) according to the manufacturer's instructions, with minor modifications as described in our previous work [7]. The resultant RNA pellet was dissolved in $20 \mu \mathrm{L}$ diethylpyrocarbonate-treated water and stored at $-80^{\circ} \mathrm{C}$. miRNA profiling analyses were performed using TLDA as previously described [8]. In brief, $3 \mu \mathrm{L}$ total RNA were added to $4.5 \mu \mathrm{L}$ of the $\mathrm{RT}$ reaction mix (Megaplex RT Primers 10X, dNTPs with dTTP $100 \mathrm{mmol}$, MultiScribe Reverse Transcriptase $50 \mathrm{U} / \mu \mathrm{L}, 10 \mathrm{X}$ RT Buffer, $\mathrm{MgCl}_{2} 25$ mmol, RNase Inhibitor $20 \mathrm{U} / \mu \mathrm{L}$ and nuclease-free water). After incubation on ice for $5 \mathrm{~min}$, reverse transcription was performed using a thermal cycle (UNO-Thermoblock, Biometra, Göttingen, Germany). miRNA profiling of 754 different human miRNAs was then performed using the TLDA (TaqMan Array Human MicroRNA A + B Cards Set v3.0) (Life Technologies, Carlsbad, CA). In order to increase the sensitivity of the TLDA, a preamplification was performed after the reverse transcription. All steps were performed using a 7900 HT Fast Real-Time PCR System (Applied Biosystems, Foster City, CA) and all reactions were performed as specified in the protocols of the manufacturer. miRNAs concentrations were presented as $\mathrm{Cq}$ values and normalized to an internal control recommended by the manufacturer on the calculated $\mathrm{Cq}$ of each miRNA $(\Delta \mathrm{Cq})$. The fold changes of miRNA expression were calculated using the eq. $2^{-\Delta \Delta \mathrm{Cq}} . \Delta \Delta \mathrm{Cq}$ was determined by subtracting the $\Delta \mathrm{Cq}$ of the normal controls from the $\Delta \mathrm{Cq}$ of the cases.

\section{RNA extraction for qRT-PCR}

Total RNA was extracted from $100 \mathrm{~mL}$ serum with a 1step phenol/chloroform purification protocol as previously described [2]. Briefly, $100 \mu \mathrm{L}$ serum was mixed with $200 \mu \mathrm{L}$ acid phenol, $200 \mu \mathrm{L}$ chloroform, and $300 \mu \mathrm{L}$ RNase-free water. To normalized the sample-to-sample variation of the RNA isolation step, an exogenous reference gene, plant miRNA MIR2911 (5'-GGCCGGGGGA CGGGCUGGGA), was added $\left(20 \mu \mathrm{L}\right.$ of $\left.10^{6} \mathrm{fmol} / \mu \mathrm{L}\right)$ to each denatured sample. The mixture was vortex-mixed vigorously and centrifuged at room temperature for 15 min. After phase separation, the aqueous layer was mixed with 2 volumes of isopropyl alcohol and 0.1 volumes of $3 \mathrm{~mol} / \mathrm{L}$ sodium acetate ( $\mathrm{pH}$ 5.3). This solution was stored at $-20^{\circ} \mathrm{C}$ for $1 \mathrm{~h}$. The RNA pellet was collected by centrifugation at $16,000 \mathrm{~g}$ for $20 \mathrm{~min}$ at $4{ }^{\circ} \mathrm{C}$. The resulting RNA pellet was washed once with 750 $\mathrm{mL} / \mathrm{L}$ ethanol and dried for $10 \mathrm{~min}$ at room temperature. Typically, after extracting total RNA from $100 \mu \mathrm{L}$ serum, the amounts of RNA in the yields were in the range of approximate $50-100 \mathrm{ng}$.
qRT-PCR

qRT-PCR assay was then conducted according to the manufacturer's instructions with a minor modification [3]. Briefly, the reverse transcription reaction was carried out in $10 \mu \mathrm{L}$ containing $2 \mu \mathrm{L}$ of extract RNA, $1 \mu \mathrm{L}$ of 10 $\mathrm{mmol} / \mathrm{L} \mathrm{dNTPs}, 0.5 \mu \mathrm{L}$ of AMV reverse transcriptase (TaKaRa), $1 \mu \mathrm{L}$ of a stem-loop RT primer (Applied Biosystems), $2 \mu \mathrm{L}$ of $5 \mathrm{X}$ reverse transcription buffer and $3.5 \mu \mathrm{L}$ of diethylpyrocarbonate (DEPC) water. For synthesis of cDNA, the reaction mixtures were incubated at $16^{\circ} \mathrm{C}$ for $30 \mathrm{~min}$, at $42^{\circ} \mathrm{C}$ for $30 \mathrm{~min}$, at $85^{\circ} \mathrm{C}$ for $5 \mathrm{~min}$, and then held at $4{ }^{\circ} \mathrm{C}$. Real-time PCR was performed $(1$ cycle of $95^{\circ} \mathrm{C}$ for $5 \mathrm{~min}$, and 40 cycles of $95^{\circ} \mathrm{C}$ for $15 \mathrm{~s}$ and $60^{\circ} \mathrm{C}$ for $1 \mathrm{~min}$ ) with Light Cycler 480 II System (Roche). The reaction was performed with a final volume of $20 \mu \mathrm{L}$ containing $1 \mu \mathrm{L}$ of $\mathrm{cDNA}, 0.3 \mu \mathrm{L}$ of Taq, $0.33 \mu \mathrm{L}$ of hydrolysis probe (Applied Biosystems), $1.2 \mu \mathrm{L}$ of $25 \mathrm{mmol} / \mathrm{L} \mathrm{MgCl}_{2}, 0.4 \mu \mathrm{L}$ of $10 \mathrm{mmol} / \mathrm{L}$ dNTPs, $2 \mu \mathrm{L}$ of 10X PCR buffer, and $14.77 \mu \mathrm{L}$ of DEPC water. All reactions, including notemplate controls, were performed in triplicate. The relative levels of miRNAs were normalized to MIR2911 and were calculated us-ing the $2^{-\Delta C q}$ method. $\Delta \mathrm{Cq}$ was calculated by subtracting the $\mathrm{Cq}$ values of MIR2911 from the average Cq values of the target miRNAs. The fold changes of miRNA expression were calculated using the eq. $2^{-\Delta \Delta \mathrm{Cq}} . \Delta \Delta \mathrm{Cq}$ was determined by subtracting the mean $\Delta \mathrm{Cq}$ of the normal controls from the mean $\Delta \mathrm{Cq}$ of the cases.

\section{Statistical analysis}

The relative expressions of miRNAs are presented as means (SEM), and other quantitative data are presented as the means (SD). Qualitative data are shown as percentages. The Kolmogorov-Smirnov test was used initially on all continuous variables to test for normal distribution. Comparisons between two groups were tested with the Independent-samples $\mathrm{T}$ test, and those between multiple groups were analyzed with the oneway ANOVA followed by the LSD test for normally distributed quantitative data with equal variance. Otherwise, the Mann-Whitney U test or Kruskal-Wallis test was used. Fisher's exact test or the chi-square test was used to compare categorical variables. Correlations between two continuous variables were performed using the Spearman rank correlation.

The prognostic value of miRNAs for predicting the development of SAP was determined using receiveroperating characteristic (ROC) curve and logistic regression analyses. The area under the ROC curve (AUC), together with the corresponding sensitivity and specificity, was used to measure the predictive accuracy. Univariate and multivariate logistic regression were used to identify independent predictors of SAP. 
Intergroup differences with $p<0.05$ were regarded as statistically significant, except for multiple comparisons after the Kruskal-Wallis test, where adjusted $p$ values of less than 0.017 (number of groups $=3$ ) or 0.008 (number of groups $=4$ ) were considered statistically significant. The statistical analyses were conducted using the SPSS 22.0 software. An optimal cutoff point of a ROC curve was calculated by MedCalc software with the maximum value of the Youden index (YI, $=1-\alpha-\beta, \alpha$, misdiagnosis rate, $\beta$, omission diagnostic rate).

\section{Results}

\section{Characteristics of the study participants}

There were 513 patients with AP hospitalized at Jinling Hospital between March 2014 and March 2015. One hundred and twenty-five patients who were admitted within $48 \mathrm{~h}$ of the onset of pain were enrolled in the present study. They were then classified into MAP $(n=45)$, MSAP $(n=42)$ and SAP $(n=38)$ groups based on the Atlanta 2012. Main clinical and demographic data are shown in Table 1 . There were no significant differences in age and sex among the 4 groups $(p>0.05)$. Significantly increased length of hospital stay, ICU admission, and length of ICU stay were observed in SAP compared with MSAP $(p<$ 0.001 ). Moreover, 27 of the 38 SAP patients, while only 2 of the 42MSAP patients required further interventions. Although the APACHE II and Ranson scores significantly differed between MSAP and SAP, none of the existing biomarkers, including CRP (C-reactive protein), could identify SAP from MSAP $(p>0.05)$.

\section{TLDA analysis}

To identify dysregulated miRNAs in patients with different severities of AP, 3 pooled serum total RNAs were examined by TLDA (Fig. 1). A miRNA was considered altered if its relative expression was at least 2 folds higher than that of the healthy control (HC) group. Of the 754 miRNAs, 395 were differentially expressed between the $\mathrm{AP}$ patients and the $\mathrm{HCs}$, among which 24 miRNAs had the same trend of alteration between the MAP patients and the SAP patients and were altered more markedly in the SAP patients than in the MAP patients (Table 2).

Table 1 Patient characteristics ${ }^{a}$

\begin{tabular}{|c|c|c|c|c|c|}
\hline & $\mathrm{HCs}(n=52)$ & $(n=45)$ & MSAP $(n=42)$ & $\operatorname{SAP}(n=38)$ & $p$ \\
\hline Age (years) & $47.4(17.1)$ & $42.3(16.9)$ & $48.1(15.8)$ & $44.6(14.7)$ & $0.835^{\mathbf{b}}$ \\
\hline Male (\% total) & $32(61.5)$ & $29(64.3)$ & $27(64.3)$ & $22(57.9)$ & $0.923^{c}$ \\
\hline Etiology (\% total) & - & - & - & - & $0.037^{c}$ \\
\hline Gallstones & - & $22(48.9)$ & $17(40.5)$ & $19(50.0)$ & - \\
\hline Hyperlipidemia & - & $15(33.3)$ & $13(31.0)$ & $18(47.4)$ & - \\
\hline Alcohol misuse & - & - & $3(7.1)$ & - & - \\
\hline Other & - & $8(17.8)$ & $12(28.6)$ & $1(2.6)$ & - \\
\hline WBC $\left(\times 10^{9} / L\right)^{d, e, f}$ & $6.0(1.5)$ & $10.6(4.0)$ & $13.0(5.5)$ & $13.4(5.5)$ & $<0.001^{\mathbf{b}}$ \\
\hline $\operatorname{CRP}(\mathrm{mg} / \mathrm{L})^{\mathrm{d}, e, f, g, h}$ & $2.7(2.4)$ & $39.5(53.6)$ & $97.3(83.1)$ & $135.0(79.3)$ & $<0.001^{\mathbf{b}}$ \\
\hline SGOT $(U / L)^{d, e, f}$ & $20.7(4.6)$ & $93.8(170.5)$ & $120.4(190.3)$ & $125.0(166.0)$ & $<0.001^{\mathbf{b}}$ \\
\hline BUN (mmol/L) & $5.0(1.0)$ & $12.1(48.0)$ & $4.8(2.1)$ & $6.8(6.5)$ & $0.245^{\mathbf{b}}$ \\
\hline Serum creatinine $(\mu \mathrm{mol} / \mathrm{L})$ & $55.7(11.1)$ & $60.7(19.0)$ & $60.6(16.7)$ & $71.7(55.8)$ & $0.492^{\mathbf{b}}$ \\
\hline Ranson score ${ }^{\mathrm{d}, \mathrm{e}, \mathrm{f}, \mathrm{g}, \mathrm{h}, \mathrm{i}}$ & $0.4(0.5)$ & $1.1(1.0)$ & $1.9(1.2)$ & $3.0(1.4)$ & $<0.001^{\mathbf{b}}$ \\
\hline APACHE $\|^{e, f, g, h}$ & $1.7(1.7)$ & $2.4(2.0)$ & $4.2(2.5)$ & $6.2(3.9)$ & $<0.001^{\mathbf{b}}$ \\
\hline Length of stay (days) ${ }^{\text {h, i }}$ & - & $9.2(3.2)$ & $10.2(4.5)$ & $18.7(10.7)$ & $<0.001^{\mathbf{b}}$ \\
\hline ICU admission (\% total) ${ }^{\mathrm{h}, \mathrm{i}}$ & - & $25(55.6)$ & $31(73.8)$ & $37(97.4)$ & $<0.001^{c}$ \\
\hline Length of ICU stay (days) ${ }^{\mathrm{h}, \mathrm{i}}$ & - & $2.2(2.8)$ & $3.7(3.7)$ & $10.7(6.7)$ & $<0.001^{\mathbf{b}}$ \\
\hline Need for intervention & - & - & 2 & 27 & - \\
\hline Surgical & - & - & - & 1 & - \\
\hline Interventional & - & - & 2 & $15^{\mathrm{j}}$ & - \\
\hline Supportive & & - & - & $21^{j}$ & - \\
\hline Mortality & - & - & - & 1 & - \\
\hline
\end{tabular}

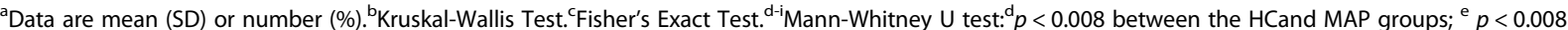
between the HC and MSAP groups; ${ }^{f} p<0.008$ between the HC and SAP groups; ${ }^{9} p<0.008$ between the MAP and MSAP groups; ${ }^{h} p<0.008$ between the MAP and SAP groups; $p<0.008$ between the MSAP and SAP groups. ${ }^{j} 10$ patients needed both interventional and supportive intervention. HC Healthy controls, MAP Mild acute pancreatitis, MSAP Moderately severe acute pancreatitis, SAP Severe acute pancreatitis, WBC White blood count, CRP C-reactive protein, SGOT Serum glutamic oxaloacetic transaminase, BUN Blood urea nitrogen, APACHE /I Acute Physiology and Chronic Health Evaluation II 
Table 2 Levels of miRNAs using TaqMan low density array

\begin{tabular}{|c|c|c|c|c|c|c|c|c|}
\hline \multirow[t]{2}{*}{ miRNAs } & \multicolumn{2}{|l|}{$\mathrm{HC}$} & \multicolumn{2}{|l|}{ MAP } & \multirow{2}{*}{$\begin{array}{l}\text { Fold } \\
\text { change } \\
\text { (MAP/HC) }\end{array}$} & \multicolumn{2}{|l|}{$\underline{\text { SAP }}$} & \multirow{2}{*}{$\begin{array}{l}\text { Fold } \\
\text { change } \\
\text { (SAP/HC) }\end{array}$} \\
\hline & $\mathrm{Cq}$ & Ave $\Delta \mathrm{Cq}$ & $\mathrm{Cq}$ & Ave $\Delta \mathrm{Cq}$ & & $\mathrm{Cq}$ & Ave $\Delta \mathrm{Cq}$ & \\
\hline miR-143 & Undetermined & 22.45 & 29.68 & 12.33 & $1.11 \times 10^{3}$ & 26.52 & 8.65 & $1.43 \times 10^{4}$ \\
\hline miR-374-5p & 30.99 & 13.44 & 28.83 & 11.48 & $3.91 \times 10^{0}$ & 24.58 & 6.71 & $1.07 \times 10^{2}$ \\
\hline miR-19a & 32.10 & 14.56 & 26.39 & 9.03 & $4.60 \times 10^{1}$ & 26.06 & 8.19 & $8.28 \times 10^{1}$ \\
\hline miR-744 & 30.99 & 13.44 & 28.04 & 10.68 & $6.78 \times 10^{0}$ & 26.10 & 8.23 & $3.72 \times 10^{1}$ \\
\hline miR-451 & 28.50 & 10.96 & 26.58 & 9.23 & $3.31 \times 10^{0}$ & 23.82 & 5.95 & $3.22 \times 10^{1}$ \\
\hline miR-200c & 30.54 & 12.99 & 28.41 & 11.06 & $3.81 \times 10^{0}$ & 27.06 & 9.19 & $1.39 \times 10^{1}$ \\
\hline miR-874 & 4.83 & -12.71 & 27.30 & 9.95 & $1.50 \times 10^{-7}$ & 29.06 & 11.19 & $6.38 \times 10^{-8}$ \\
\hline miR-216a & 20.98 & 3.44 & 32.40 & 15.05 & $3.19 \times 10^{-4}$ & Undetermined & 22.13 & $2.36 \times 10^{-6}$ \\
\hline miR-381 & 22.34 & 4.79 & 30.04 & 12.69 & $4.21 \times 10^{-3}$ & Undetermined & 22.13 & $6.05 \times 10^{-6}$ \\
\hline miR-518f & 23.08 & 5.53 & 25.58 & 8.23 & $1.54 \times 10^{-1}$ & Undetermined & 22.13 & $1.01 \times 10^{-5}$ \\
\hline miR-219 & 25.42 & 7.87 & 28.01 & 10.66 & $1.45 \times 10^{-1}$ & Undetermined & 22.13 & $5.10 \times 10^{-5}$ \\
\hline miR-136 & 26.70 & 9.15 & 34.07 & 16.72 & $5.28 \times 10^{-3}$ & Undetermined & 22.13 & $1.24 \times 10^{-4}$ \\
\hline miR-184 & 27.12 & 9.57 & 28.99 & 11.64 & $2.39 \times 10^{-1}$ & Undetermined & 22.13 & $1.66 \times 10^{-4}$ \\
\hline miR-367 & 27.40 & 9.85 & 32.59 & 15.24 & $2.39 \times 10^{-2}$ & Undetermined & 22.13 & $2.01 \times 10^{-4}$ \\
\hline miR-147 & 29.00 & 11.45 & 37.87 & 20.52 & $1.87 \times 10^{-3}$ & Undetermined & 22.13 & $6.11 \times 10^{-4}$ \\
\hline miR-331-5p & 29.77 & 12.23 & 30.84 & 13.48 & $4.19 \times 10^{-1}$ & Undetermined & 22.13 & $1.04 \times 10^{-3}$ \\
\hline miR-618 & 23.01 & 5.46 & 26.72 & 9.37 & $6.65 \times 10^{-2}$ & 31.66 & 13.79 & $3.11 \times 10^{-3}$ \\
\hline miR-205 & 26.85 & 9.30 & 29.96 & 12.61 & $1.01 \times 10^{-1}$ & 34.31 & 16.44 & $7.09 \times 10^{-3}$ \\
\hline miR-342-5p & 29.07 & 11.53 & 32.52 & 15.17 & $8.01 \times 10^{-1}$ & 33.85 & 15.98 & $4.58 \times 10^{-2}$ \\
\hline miR-139-3p & 26.02 & 8.47 & 26.85 & 9.50 & $4.91 \times 10^{-1}$ & 30.23 & 12.36 & $6.74 \times 10^{-2}$ \\
\hline miR-194 & 25.01 & 7.46 & 26.82 & 9.47 & $2.48 \times 10^{-1}$ & 28.68 & 10.81 & $9.78 \times 10^{-2}$ \\
\hline miR-491 & 24.69 & 7.14 & 26.69 & 9.34 & $2.19 \times 10^{-1}$ & 27.88 & 10.01 & $1.37 \times 10^{-1}$ \\
\hline let-7b & 20.71 & 3.17 & 23.18 & 5.83 & $1.58 \times 10^{-1}$ & 23.87 & 6.00 & $1.40 \times 10^{-1}$ \\
\hline miR-433 & 26.82 & 9.27 & 28.48 & 11.12 & $2.77 \times 10^{-1}$ & 29.92 & 12.05 & $1.46 \times 10^{-1}$ \\
\hline
\end{tabular}

Undetermined data were referred to as 40

\section{Confirmation of miRNAs expression by qRT-PCR}

Although TLDA is based on qRT-PCR assay, it was performed on only one pooled sample for each group. Consequently, the array results must be validated by a qRT-PCR assay in individual samples. Therefore, 12 miRNAs, including miR-143, miR-374-5p, miR-19a, miR-744, miR-451, miR-200c, miR-874, miR-216a, miR381, miR-518f, miR-219, and miR-136, were selected for further evaluation by qRT-PCR in $20 \mathrm{HCs}, 20$ patients with MAP, 20 patients with MSAP, and 20 patients with SAP (referred to as the training set). Consistent with TLDA, miR-19a, miR-143, miR-374-5p, miR-200c and miR-451 levels increased significantly in the MSAP and SAP groups compared with the HCs $(p<0.008)$. Moreover, miR-143 and miR-374-5p levels were significantly elevated in the SAP group compared with the MAP group $(p<0.008)$.

Three miRNAs (miR-19a, miR-143 and miR-374-5p) were selected to establish an expression signature because these miRNAs has a $p$ value $\leq 0.001$ and a fold change $\geq 2$ (comparing all AP patients with the $\mathrm{HCs}$ ) (Supplemental Table S1). The three miRNAs were examined using qRT-PCR in another, larger cohort consisting of 25 MAP, 22 MSAP, 18 SAP patients and 32 matched HCs. As shown in Supplemental Table S2, all three miRNAs showed the same trend with the training set and had a higher serum level in the SAP patients than in the MAP patients $(p<0.008)$.

As shown in Fig. 2, when the training and validation sets were combined, miR-19a, miR-143 and miR-374-5p increased with disease severity. Statistical analyses revealed that the expression of the three miRNAs were significantly different among all paired groups $(p<0.05)$.

\section{Diagnostic accuracy analysis of the selected serum miRNAs}

As miR-19a, miR-143 and miR-374-5p levels were higher in the SAP group than in the HCs, MAP and MSAP group, they might identify SAP patients from other patients. ROC analyses were performed to determine the 


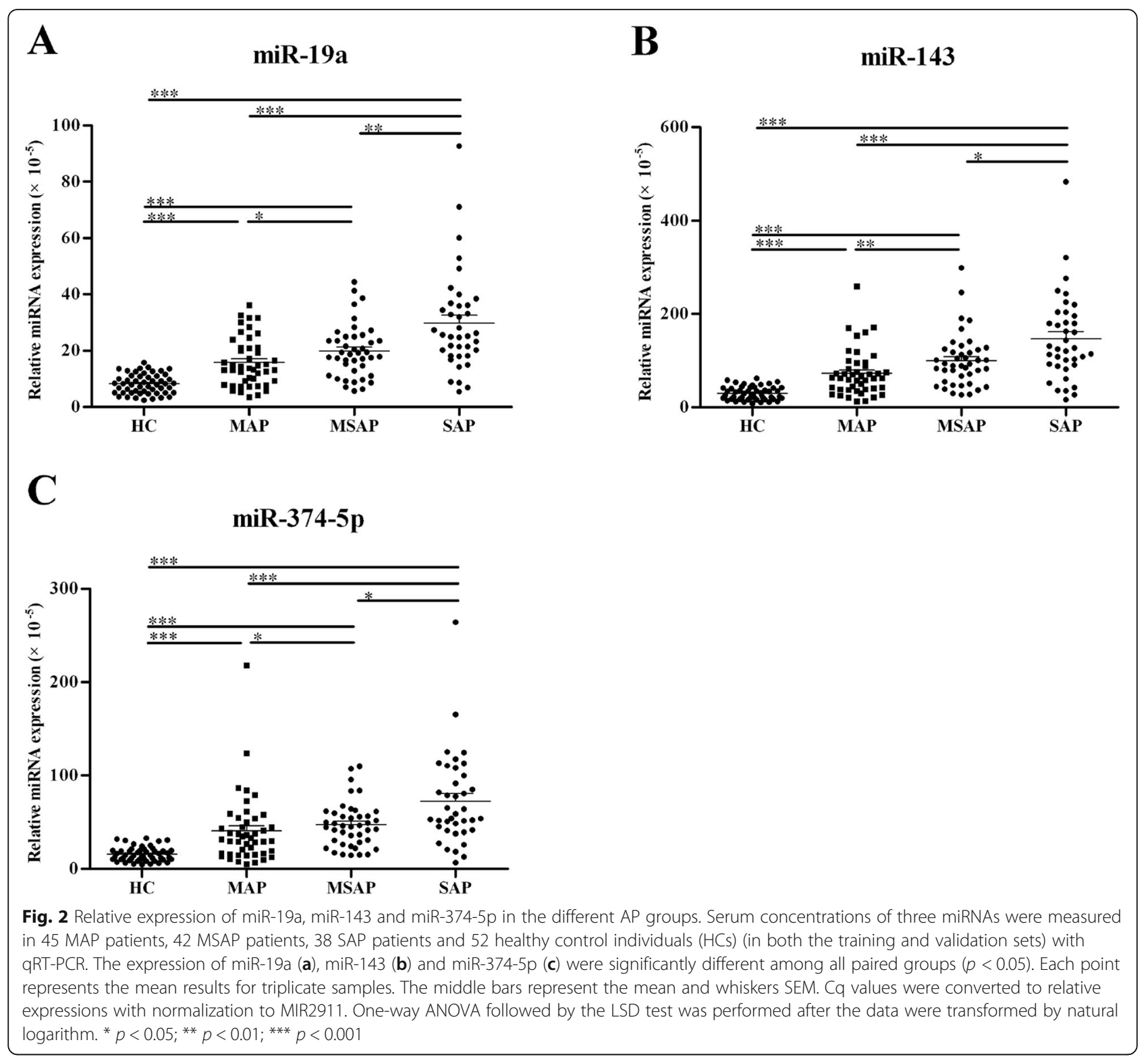

accuracy. The analyses were initially confined between the HC and SAP groups. As shown in Fig. 3 and Supplemental Table S3, the AUCs for miR-19a, miR-143 and miR-374-5p were 0.943 (95\% CI, 0.874-0.981), 0.940 (95\% CI, 0.870-0.979) and 0.941 (95\% CI, 0.870-0.980), respectively. Likewise, when MAP was used as the reference, SAP could also be distinguished by miR-19a, miR143 and miR-374-5p with AUCs of 0.781 (95\% CI, $0.676-0.864), 0.782$ (95\% CI, 0.678-0.866) and 0.754 (95\% CI, 0.647-0.842), respectively. Because the majority of patients with MAP recover uneventfully, differentiating SAP from MSAP was crucial for decision making. As shown in Fig. 3 and Supplemental Table S3, all three miRNAs could identify SAP from MSAP, whereas CRP could not differentiate SAP from MSAP $(p=0.056)$. The
AUCs for 3 selected miRNAs were as follows: miR-19a, 0.686 (95\% CI 0.573-0.785); miR-143, 0.671 (95\% CI 0.559-0.773); miR-374-5p, 0.670 (95\% CI 0.556-0.772), comparable to the APACHE II $(0.655,95 \%$ CI $0.540-$ $0.758)$ and Ranson $(0.762$, 95\% CI $0.653-0.850)$ scores. Using the optimal cutoff value, we obtained the following sensitivity and specificity values: 57.89 and $73.81 \%$ for miR-19a; 44.74 and $88.10 \%$ for miR-143; 47.34 and 83.33\% for miR-374-5p; 71.05 and $59.52 \%$ for CRP; 55.26 and $71.43 \%$ for APACHE II; 44.74 and $92.86 \%$ for Ranson respectively. When all AP patients were analyzed, the AUCs for the 3 miRNAs ranged from 0.714 to 0.735 (95\% CI, 0.626-0.810), which was higher than those for the MSAP or SAP patients (Supplemental Table S3). Besides, three miRNAs correlated well to 

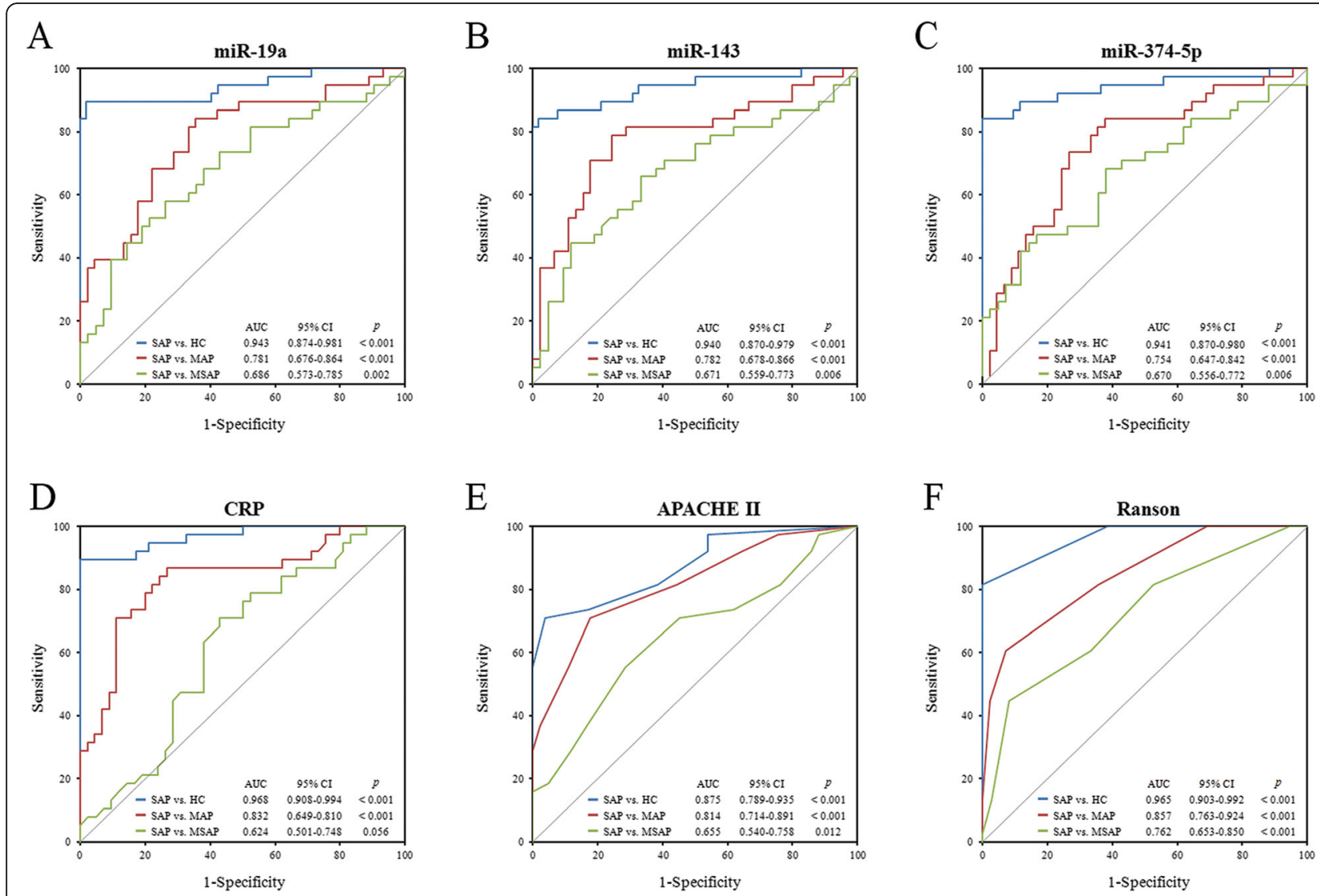

Fig. 3 ROC curve analysis for the ability of serum biomarkers to predict the development of SAP $(n=38)$ from $\operatorname{HCS}(n=52), \operatorname{MAP}(n=45)$ and MSAP $(n=42)$. MiR-19a (a), miR-143 (b), miR-374-5p (c), as well as APACHE II (e) and Ranson scores (f), could identify SAP patients from HC, MAP, especially MSAP, whereas CRP $(\mathbf{d})$, could not differentiate SAP from MSAP $(p=0.056)$. The AUCs for 3 selected miRNAs were comparable to the APACHE II and Ranson scores

APACHE II and Ranson (Supplemental Table S4), indicating their potential in prediction of SAP.

\section{Selected miRNAs to predict the development of SAP}

As three miRNAs could distinguish the SAP patients from the HCs and the MAP and MSAP patients, they may be potentially applied to predict the development of SAP. Subsequently, univariate and multivariate logistic regression analyses were performed to identify distinctive miRNAs associated with SAP in different cohorts. After adjusting for age, gender, and confounding factors and using the control group as the reference category when the SAP/MSAP/MAP or control group was treated as a dependent four-category variable, serum miR-19a $\left(\right.$ cutoff $\left.=14 \times 10^{-5}\right)$ and miR-143 (cutoff $\left.=58 \times 10^{-5}\right)$ were independently correlated with SAP development. Furthermore, with different cutoff values in different cohorts, miR-19a, miR-143 and miR-374-5p were found to be independently associated with SAP development when the MAP group, MSAP group or combination of the two groups was treated as the reference category. When multivariate logistic regression was conducted, only miR-143 and CRP showed independent association with the presence of SAP when MAP, MSAP or combined MAP and MSAP was treated as the reference category. When MSAP was the reference, the OR values of miR-143 (cutoff $\left.=141 \times 10^{-5}\right)$ and CRP (cutoff $=114$ ) were 6.8 (95\% CI 2.0-22.7, $p=0.002)$ and 3.8 (95\% CI $1.4-10.6, p=0.011$ ), respectively (Table 3 ). The combination of miR-143 and CRP improved the specificity to $100 \%$ and the AUC to 0.728 (95\% CI $0.617-0.822$ ), which was comparable to Ranson score (specificity, 92.86\%; AUC $=0.762$, 95\% CI 0.653-0.850) (Supplemental Table S3). When all AP patients were analyzed, logistic regression analysis showed that miR-143 and CRP with cutoffs of $101 \times 10^{-5}$ and $114 \times 10^{-5}$ respectively, were independent predictors of SAP with OR values of 4.5 (95\% CI $1.8-10.9, p=0.001)$ and 5.1 (95\% CI 2.1$12.4, p<0.001$ ), respectively (Table 3 ). Combining miR143 and CRP also improved the specificity $(89.66 \%)$ and AUC (0.786, 95\% CI 0.703-0.854) of prediction for SAP, similar to Ranson score (specificity, 82.76\%; AUC= 0.811, 95\% CI 0.731-0.876) (Supplemental Table S3). In addition, miR-143 also showed independent association 
Table 3 Logistic regression analyses of biomarkers in predicting severe acute pancreatitis

\begin{tabular}{|c|c|c|c|c|c|}
\hline \multirow[t]{2}{*}{ Variables } & \multirow[t]{2}{*}{ Cutoff } & \multicolumn{2}{|l|}{ Univariate analyses } & \multicolumn{2}{|c|}{ Multivariate analyses } \\
\hline & & $\mathrm{OR}(95 \% \mathrm{Cl})$ & $p$ & $\overline{\mathrm{OR}}(95 \% \mathrm{Cl})$ & $p$ \\
\hline \multicolumn{6}{|l|}{ Model $1^{a}$} \\
\hline miR-19a $\left(\times 10^{-5}\right)$ & 14 & $433.5(46.4-4047.1)$ & $<0.001$ & $69.2(4.8-992.2)$ & 0.002 \\
\hline $\operatorname{miR}-143\left(\times 10^{-5}\right)$ & 58 & $272.0(31.3-2364.8)$ & $<0.001$ & $13.5(0.7-244.8)$ & 0.078 \\
\hline $\operatorname{miR}-374-5 p\left(\times 10^{-5}\right)$ & 33 & NA & 0.997 & & \\
\hline CRP (mg/L) & 8 & NA & 0.997 & & \\
\hline \multicolumn{6}{|l|}{ Model $2^{b}$} \\
\hline $\operatorname{miR}-19 a\left(\times 10^{-5}\right)$ & 17 & $9.7(3.3-28.0)$ & $<0.001$ & & \\
\hline $\operatorname{miR}-143\left(\times 10^{-5}\right)$ & 88 & $11.6(4.1-32.6)$ & $<0.001$ & $18.1(4.7-69.3)$ & $<0.001$ \\
\hline miR-374-5p $\left(\times 10^{-5}\right)$ & 45 & $7.7(2.9-20.5)$ & $<0.001$ & & \\
\hline CRP (mg/L) & 36 & $18.2(5.8-57.3)$ & $<0.001$ & $11.5(3.2-41.9)$ & $<0.001$ \\
\hline \multicolumn{6}{|l|}{ Model $3^{c}$} \\
\hline miR-19a $\left(\times 10^{-5}\right)$ & 24 & $6.0(1.9-18.6)$ & 0.002 & & \\
\hline $\operatorname{miR}-143\left(\times 10^{-5}\right)$ & 142 & $3.3(1.3-8.3)$ & 0.012 & $6.8(2.0-22.7)$ & 0.002 \\
\hline miR-374-5p $\left(\times 10^{-5}\right)$ & 63 & $3.9(1.5-9.9)$ & 0.005 & & \\
\hline CRP (mg/L) & 114 & $4.5(1.6-12.6)$ & 0.004 & $3.8(1.4-10.6)$ & 0.011 \\
\hline \multicolumn{6}{|l|}{ Model $4^{d}$} \\
\hline miR-19a $\left(\times 10^{-5}\right)$ & 18 & $5.8(2.5-13.3)$ & $<0.001$ & & \\
\hline $\operatorname{miR}-143\left(\times 10^{-5}\right)$ & 101 & $6.4(2.8-15.0)$ & $<0.001$ & $4.5(1.8-10.9)$ & 0.001 \\
\hline $\operatorname{miR}-374-5 p\left(\times 10^{-5}\right)$ & 50 & $5.7(2.3-14.4)$ & $<0.001$ & & \\
\hline CRP (mg/L) & 114 & $4.6(2.0-10.4)$ & $<0.001$ & $5.1(2.1-12.4)$ & $<0.001$ \\
\hline
\end{tabular}

${ }^{a}$ the reference category was the $\mathrm{HC} ;{ }^{b}$ the reference category was MAP; ${ }^{c}$ the reference category was MSAP; ${ }^{d}$ the reference category was MAP and MSAP. OR Odds ratio, $\mathrm{Cl}$ Confidence intervals, NA Not assessed, as at least one cell has zero frequencies

with the presence of local complications $(\mathrm{OR}=6.2,95 \%$ CI 1.1-35.3, $p=0.038$ ) (Supplemental Table S5).

Moreover, miR-19a, miR-143 and miR-374-5p showed a positive correlation with $\mathrm{CRP}$ at admission $(\mathrm{R}=0.50$, 0.51 and 0.49 , respectively, $p<0.001$ ) (Supplemental Fig. S1), but they identified partially different patients at risk as miR143 and CRP were independently associated with SAP development. Taken together, these analyses revealed that miR-143 may be a better biomarker than CRP for predicting SAP among patients with MSAP and SAP. Moreover, both among all patients and among those with MSAP and SAP, combining miR-143 and CRP improved the accuracy to that of the more complicated scoring systems for early prediction of SAP.

\section{Dynamic expression of the selected serum miRNAs at different time points}

In addition, the dynamic expressions of miR-19a, miR-143 and miR-374-5p were explored. Among all the patients, we collected 21 serum samples (10 MAP, 6 MSAP, 5 SAP) on day 2, 47 samples (14 MAP, 16 MSAP, 17 SAP) on day 3, 52 samples (17 MAP, 16 MSAP, 19 SAP) on day 7, 13 samples (1 MAP, 8 MSAP, 4 SAP) on day 14 and 11 samples (1 MAP, 4 MSAP, 6 SAP) on day 21respectively (Fig. 4). Data analyses were performed on SAP, non-SAP (including MAP and MSAP) and HC groups. As shown in Fig. 4, the levels of miR-19a, miR-143 and miR-374-5p were higher in the SAP patients at all time points through 21 days after admission than in the HCs $(p<0.017)$ and the non-SAP patients. Of the three miRNAs, miR-19a in the SAP group increased gradually and achieved its peak at day 14, then declined at day 21 . The trend was similar in the non-SAP group except for day 2, when the expression decreased markedly. However, miR-143 in the SAP group reached its peak at approximately day 7 and declined from day 14, whereas in the non-SAP group, the fluctuation was not obvious. miR-374-5p showed a trend similar to that of miR-19a, which reached its peak at day 14 , then declined at day 21. Notably, miR-374-5p had a significantly higher levels in the SAP and non-SAP patients from day 1 to day $3(p<0.017)$, suggesting it may more sensitively predict SAP. At day 21 , all three miRNAs declined but still remained higher in the SAP and nonSAP patients than in the HCs $(p<0.017$ for each), except for miR-19a in the non-SAP group $(p=0.025)$.

Furthermore, as shown in Supplemental Table S6, using the optimal cutoff value, the peak levels of the three miRNAs were significantly correlated with the presence of local complications $(p<0.001$ for each). Moreover, patients with peak expressions of miR-19a 
A

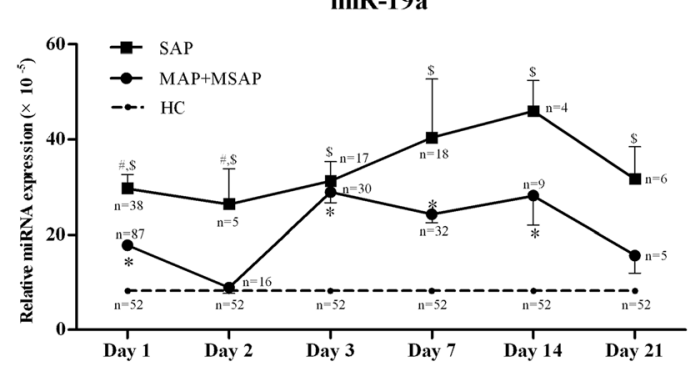

C

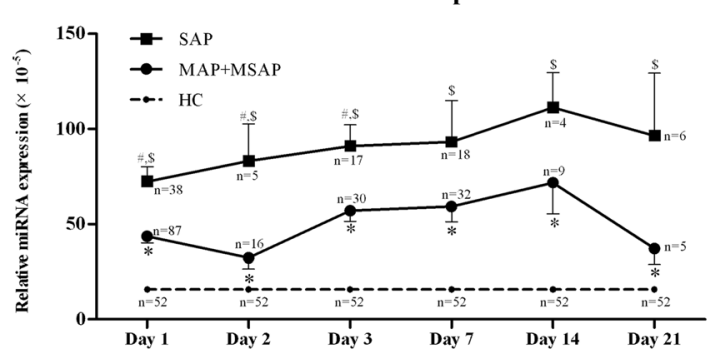

B

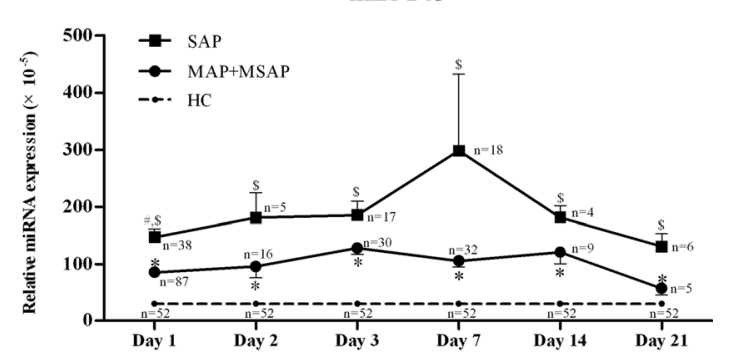

Fig. 4 Expression levels of miR-19a, miR-143 and miR-374-5p at different time points during AP. The levels of 3 miRNAs in the SAP and non-SAP (MAP and MSAP) groups were measured on admission and at different time points thereafter, as indicated. a miR-19a in SAP peaked at day 14 and declined at day 21. The trend was similar in the non-SAP group, except for day 2. b miR-143 in SAP peaked at approximately day 7 and declined from day 14. The fluctuation was not obvious in the non-SAP groups. $\mathbf{c}$ miR-374-5p showed a trend similar to that of miR-19a and was different between SAP and non-SAP from day 1 to day 3. Cq values were converted to relative expressions with normalization to MIR2911. The Mann-Whitney $U$ test was performed subsequent to the Kruskal-Wallis test to compare the differences among groups. $n$, number of patients in the subgroups. ${ }^{\#} p<0.017$ between SAP and non-SAP patients; ${ }^{\$} p<0.017$ between SAP and HC; ${ }^{*} p<0.017$ between non-SAP and HC

and miR-374-5p at day 14 were more likely to be treated with interventions ( $p=0.003$ and 0.000 respectively).

\section{Discussion}

In the present study, we focused on the serum miRNA profile in patients with AP and in the MAP, MSAP and SAP subgroups based on Atlanta 2012. Our data revealed that AP patients had higher circulating levels of miR-19a, miR-143 and miR-374-5p at admission than the HCs. The level of serum miR-19a, miR-143 and miR-374-5p at admission could differentiate the SAP patients from the HCs, MAP and MSAP patients and correlated well with APACHE II, Ranson score and CRP level. Moreover, miR-143 and CRP were independent factors in a multivariable logistic regression model for the development of SAP among MSAP and SAP patients and among all AP patients. Combining the two variables (miR-143 and CRP) improved the predictive performance to a level comparable with the APACHE II and Ranson score. Thus, we may identify patients with SAP within $6 \mathrm{~h}$ of admission by using three miRNAs as novel biomarkers.

Several studies have investigated circulating miRNAs in AP patients. An overexpression of miR-155 has been reported to be correlated with SAP [9], but it was also demonstrated to be inversely correlated with SAP in another study [10]. Lee $\mathrm{Hb}$ et al. have shown that miR216a and miR-375 could predict the degree of pancreatic injury in dog model [11].Furthermore, some studies focused on serum miRNAs profiles of SAP patients with a specific organ failure [12, 13]. Notably, the miRNAs profile in the present study was quite different from those reported by others [6, 9-13]. One possible reason is that the classifications of enrolled patients were different. Another reason might be the different normalization strategy applied in our study. In the previous studies, U6 or miR-16 was used as the internal reference gene. While in our study, we normalized miRNAs concentration to an exogenous spiking small RNA, named MIR2911, and evidence has demonstrated that an external spike-in synthetic oligonucleotide performs better for accurately determining the serum miRNA abundance in certain diseases [14]

Why serum levels of miRNAs changes after pancreatic injury is still not well understood. In the dynamic process of AP, there are two overlapping phases: early and late [3]. During the early phase, the inflammatory reaction initially occurs in the pancreas. Subsequently, 
inflammatory cytokines are released and cause systemic inflammatory response syndrome (SIRS). When SIRS is persistent, there is an increased risk of developing organ failure, which is recognized as the dominant factor of SAP [15]. In the present study, all three miRNAs showed positive correlations to CRP and their levels peaked at 1 to 2 weeks and decreased slowly thereafter, suggesting that they might be involved in the process of SIRS and be related to the inflammatory status of AP patients. MiR-19a and miR-374-5p have been demonstrated to be related to inflammation. MiR-19a, belonging to the cluster mir-17-92, has been proved to be regulated by the $N F-\kappa B$ p65 subunit and has a positive effect on NF- $\kappa B$ signaling $[16,17]$. NF- $\mathrm{kB}$ plays a key role in the pathogenesis of AP [18], suggesting that miR-19a is related to the initiation and cascade of inflammation in AP. Besides, using TargetScan, miR-19a was found to potentially regulate some genes related with inflammatory cascade, such as serum/glucocorticoid regulated kinase 1 . Owen et al. reported that the miR-374 family of miRNAs is linked to increased IL-10 expression in trauma patients, indicating its potential role in acute injury [19]. MiR-374-5p, miR19a and miR-143 could be detected in pancreatic tissues and are reported to take part in the development of pancreatic cancer [20-22]. It has been demonstrated that miR-143 could promote apoptosis and be involved in a regulatory pathway in KRAS mutant pancreatic cancers $[23,24]$. Moreover, miR-143 expression was shown to be pancreas and liver specific, suggesting its potential role in AP [25]. Further study is necessary to elucidate the roles of miR-19a, miR-143 and miR-374-5p in the pathogenesis of AP and development of SAP.

\section{Conclusions}

In the present study, we report the circulating miRNAs profile in patients with AP and subgroups of AP according to Atlanta 2012. Our results indicate that the serum levels of miR-19a, miR-143 and miR-374-5p at admission is higher in the AP patients than the healthy controls and correlate positively with AP severity. An expression signature of three miRNAs may identify SAP patients from the healthy controls, MAP patients and MSAP patients. Furthermore, a combination of miR-143 and CRP has the potential to replace the more complicated scoring systems.

\section{Supplementary information}

Supplementary information accompanies this paper at https://doi.org/10. 1186/s41544-020-00048-z.

\section{Additional file 1.}

\section{Abbreviations}

miRNAs: microRNAs; AP: Acute pancreatitis; MAP: Mild AP; MSAP: Moderate severe AP; SAP: Severe AP; TLDA: TaqMan low density array; HCs: Healthy controls; qRT-PCR: quantitative real-time polymerase chain reaction;"
ROC: Receiver operating characteristic; AUC: Area under ROC; POF: Persistent organ failure; ICU: Intensive care unit; LOS: ICU length of stay

\section{Acknowledgements}

Not applicable.

\section{Authors' contributions}

Study concept and design: Yu-Ping Zhang, Lei Ye, Chun-Ni Zhang, Chen-Yu Zhang, Fang-Yu Wang; Acquisition of data: Yu-Ping Zhang, Lei Ye, Na Yu, Lin Wu; Analysis and interpretation of data: Yu-Ping Zhang, Lei Ye, Cheng Wang; Drafting of the manuscript: Yu-Ping Zhang; Critical revision of the manuscript for important intellectual content: Yu-Ping Zhang, Lei Ye, Cheng Wang, Chun-Ni Zhang, Chen-Yu Zhang, Fang-Yu Wang; Study supervision: Chun-Ni Zhang, Chen-Yu Zhang, Fang-Yu Wang; Guarantor of the article: Fang-Yu Wang. All authors approved the final version of the manuscript.

\section{Funding}

The work was supported by National Natural Science Foundation of China (NO.81570506). The institution didn't participate in any process of the study, including the design of the study and collection, analysis, interpretation of data and in writing the manuscript.

\section{Availability of data and materials}

The datasets generated during the study are available from the corresponding author on reasonable request.

\section{Ethics approval and consent to participate}

The study was approved by the Medical Ethics Committee of the Jinling Hospital. The consent to participate was waived.

\section{Consent for publication}

Not applicable.

\section{Competing interests}

All authors disclose no conflicts.

\section{Author details}

'Department of Endoscopy, The First Affiliated Hospital of Shandong First Medical University, Jinan 250000, China. ${ }^{2}$ Department of Gastroenterology and Hepatology, Jinling Hospital, Medical School of Nanjing University, \#305 East Zhongshan Road, Nanjing 210002, China. ${ }^{3}$ Jiangsu Provincial Key Laboratory of Geriatrics, Department of Geriatrics, the First Affiliated Hospital with Nanjing Medical University, Nanjing, Jiangsu Province, China.

${ }^{4}$ Department of Clinical Laboratory, Jinling Hospital, \#305 East Zhongshan Road, Nanjing 210002, China. ${ }^{5}$ State Key Laboratory of Pharmaceutical Biotechnology, Nanjing Advanced Institute for Life Sciences (NAILS), Nanjing University School of Life Sciences, Jiangsu Engineering Research Center for microRNA Biology and Biotechnology, \#163XianlinRoad, Nanjing 210002, China.

Received: 8 January 2020 Accepted: 29 April 2020

Published online: 30 July 2020

\section{References}

1. Yadav D, Lowenfels AB. The epidemiology of pancreatitis and pancreatic cancer. Gastroenterology. 2013;144:1252-61.

2. Whitcomb DC, Muddana $V$, Langmead CJ, et al. Angiopoietin-2, a regulator of vascular permeability in inflammation, is associated with persistent organ failure in patients with acute pancreatitis from the United States and Germany. Am J Gastroenterol. 2010;105:2287-92.

3. Banks PA, Bollen TL, Dervenis C, et al. Classification of acute pancreatitis-2012: revision of the Atlanta classification and definitions by international consensus. Gut. 2013;62:102-11.

4. Meng S, Wang $H$, Xue D, Zhang W. Screening and validation of differentially expressed extracellular miRNAs in acute pancreatitis. Mol Med Rep. 2017;16: $6412-8$

5. Zhang XX, Deng LH, Chen WW, Shi N, Jin T, Lin ZQ, et al. Circulating microRNA 216 as a marker for the early identification of severe acute pancreatitis. Am J Med Sci. 2017;353:178-86. 
6. Lu P, Wang F, Wu J, et al. Elevated serum miR-7, miR-9, miR-122, and miR141 are noninvasive biomarkers of acute pancreatitis. Dis Markers. 2017; 2017:7293459

7. Zhang $C$, Wang $C$, Chen $X$, et al. Expression profile of microRNAs in serum: a fingerprint for esophageal squamous cell carcinoma. Clin Chem. Dec 2010; 56(12):1871-9.

8. Wu C, Wang C, Guan X, et al. Diagnostic and prognostic implications of a serum miRNA panel in Oesophageal squamous cell carcinoma. PLoS One. 2014;9(3):e92292.

9. Wang D, Tang M, Zong P, Liu H, Zhang T, Liu Y, et al. MiRNA-155 regulates the Th17/Treg ratio by targeting SOCS1 in severe acute pancreatitis. Front Physiol. 2018;9:686.

10. Liu S, Zou H, Wang Y, Duan X, Chen C, Cheng W, et al. miR-155-5p is Negatively Associated with Acute Pancreatitis and Inversely Regulates Pancreatic Acinar Cell Progression by Targeting Rela and Traf3. Cell Physiol Biochem. 2018;51:1584-99.

11. Lee HB, Park HK, Choi HJ, Lee S, Lee SJ, Lee JY, et al. Evaluation of Circulating MicroRNA Biomarkers in the Acute Pancreatic Injury Dog Model. Int J Mol Sci. 2018;19:E3048.

12. Shi N, Deng L, Chen W, Zhang X, Luo R, Jin T, et al. Is MicroRNA-127 a novel biomarker for acute pancreatitis with lung injury? Dis Markers. 2017;2017: 1204295.

13. Wu XM, Ji KQ, Wang HY, Zhao Y, Jia J, Gao XP. MicroRNA-339-3p alleviates inflammation and edema and suppresses pulmonary microvascular endothelial cell apoptosis in mice with severe acute pancreatitis-associated acute lung injury by regulating Anxa3 via the Akt/mTOR signaling pathway. J Cell Biochem. 2018;119:6704-14.

14. Roberts TC, Coenen-Stass AM, Wood MJ. Assessment of RT-qPCR normalization strategies for accurate quantification of extracellular microRNAs in murine serum. PLoS One. 2014;9:e89237.

15. Peng YS, Chen YC, Tian YC, et al. Serum levels of apolipoprotein A-l and high-density lipoprotein can predict organ failure in acute pancreatitis. Crit Care. 2015;19:88

16. Zhou R, Hu G, Gong AY, Chen XM. Binding of NF-kappaB p65 subunit to the promoter elements is involved in LPS-induced transactivation of miRNA genes in human biliary epithelial cells. Nucleic Acids Res. 2010;3:3222-32.

17. Gantier MP, Stunden HJ, McCoy CE, et al. A miR-19 regulon that controls NF-kappaB signaling. Nucleic Acids Res. 2012;40:8048-58.

18. Zhao S, Yang J, Liu T, Zeng J, Mi L, Xiang K. Dexamethasone inhibits NFKBp65 and HMGB1 expression in the pancreas of rats with severe acute pancreatitis. Mol Med Rep. 2018;18:5345-52.

19. Owen HC, Torrance HDT, Barnes MR, et al. The role of Micrornas in the development of hospital acquired infection in Polytrauma patients. Intensive Care Med Exp. 2015;3(Suppl 1):A35.

20. Debernardi S, Massat NJ, Radon TP, et al. Noninvasive urinary miRNA biomarkers for early detection of pancreatic adenocarcinoma. Am J Cancer Res. 2015;5:3455-66.

21. Zou X, Wei J, Huang Z, et al. Identification of a six-miRNA panel in serum benefiting pancreatic cancer diagnosis. Cancer Med. 2019;8:2810-22. https:// doi.org/10.1002/cam4.2145.

22. Ma L, Shao Z, Zhao Y. MicroRNA-374a promotes pancreatic cancer cell proliferation and epithelial to mesenchymal transition by targeting SRCIN1. Pathol Res Pract. 2019;215:152382

23. Kent OA, Chivukula RR, Mullendore M, Wentzel EA, Feldmann G, Lee KH, Liu S, Leach SD, Maitra A, Mendell JT. Repression of the miR-143/145 cluster by oncogenic Ras initiates a tumor-promoting feed-forward pathway. Genes Dev. 2010;24:2754-9.

24. Zhang H, Cai X, Wang Y, Tang H, Tong D, Ji F. microRNA-143, downregulated in osteosarcoma, promotes apoptosis and suppresses tumorigenicity by targeting Bcl-2. Oncol Rep. 2010;24:1363-9.

25. Zhu H, Leung SW. Identification of microRNA biomarkers in type 2 diabetes: a meta-analysis of controlled profiling studies. Diabetologia. 2015;58:900-11.

\section{Publisher's Note}

Springer Nature remains neutral with regard to jurisdictional claims in published maps and institutional affiliations.

Ready to submit your research? Choose BMC and benefit from:

- fast, convenient online submission

- thorough peer review by experienced researchers in your field

- rapid publication on acceptance

- support for research data, including large and complex data types

- gold Open Access which fosters wider collaboration and increased citations

- maximum visibility for your research: over $100 \mathrm{M}$ website views per year

At $\mathrm{BMC}$, research is always in progress.

Learn more biomedcentral.com/submissions 